\title{
Neuroimaging and Neuropathologic Findings in AIDS Patient with Cytomegalovirus Infection
}

\author{
Shinichiro Nagamitsu, Sayuri Okabayashi, Shunichi Dai, Yousuke Morimitsu*, \\ Tatsuo Murakami, Toyojiro Matsuishi, Manabu Motizuki** and Hirohisa Kato
}

\begin{abstract}
This 21-year-old male with hemophilia A developed cytomegalovirus (CMV) retinitis associated with acquired immunodeficiency syndrome (AIDS). He had a history of numerous blood transfusions. Serum antibody titers became positive for human immunodeficiency virus (HIV), when the patient was 18 years of age. Three years later, he developed CMV retinitis due to his immunosuppression. Ganciclovir (DENOSINE ${ }^{\circledR}$, TANABE SEIYAKU CO., LTD., Osaka, Japan) therapy given for 4 weeks produced a marked improvement in the ocular fundal findings, but the neurologic signs and symptoms, including headache, hypoesthesia, disorientation, and dementia became worse. T2-weighted magnetic resonance imaging (MRI) demonstrated a diffuse high intensity area in the periventricular white matter and small focal or patchy lesions in the hippocampus, basal ganglia, midbrain, medulla oblongata and the nucleus dentatus. The patient died of HIV encephalopathy and CMV infection. Characteristic CMV intranuclear inclusion bodies were observed histologically in most sites of the brain including the hippocampus, white matter, basal ganglia, midbrain, medulla oblongata, nucleus dentatus and the retina. Infiltration by monocyte-macrophage and multinucleated giant cells, which are characteristic of HIV encephalopathy, were observed in the periventricular white matter and the hippocampus. In this patient, the neuroimaging findings were compatible with the neuropathologic observations. MR imaging proved useful in detecting the central nervous system (CNS) lesions of AIDS and CMV infection.
\end{abstract}

(Internal Medicine 33: 158-162, 1994)

Key words: cytomegalovirus, retinitis, AIDS, opportunistic infection, MRI

\section{Introduction}

In Japan, about 2,500 patients are infected with human immunodeficiency virus (HIV) (1). Compared to acquired immunodeficiency syndrome (AIDS) patients in the U.S.A. and Europe, there is a high proportion of hemophilia patients in Japan (1). Although there are many reports concerning the neuropathology of AIDS in other countries, the number of such studies in Japan is limited (2). Recent advances in neuroimaging by CT-scan and magnetic resonance imaging (MRI) permit a noninvasive recognition of central nervous system (CNS) lesions. We present the case of a 22-year-old male hemophiliac with AIDS encephalopathy and cytomegalovirus (CMV) infection. The diagnostic utility of MRI is compared with that obtained by neuropathologic methods.

\section{Case Report}

This 22-year-old Japanese male was diagnosed as having hemophilia A at age 4, and had a history of numerous blood transfusions. He became seropositive for human immunodeficiency virus (HIV) antibody at age 18, and his immunocompetence gradually declined. He was frequently hospitalized with oral candidiasis and pneumonia. Zidovudine (RETROVIR ${ }^{\circledR}$, NIPPON WELLCOME K.K., Osaka, Japan) administration was begun in February 1990. When the patient was 21-years-old, he complained of impairment of the visual field and of ocular "floaters". Ophthalmoscopic findings led to a diagnosis of bilateral cytomegalovirus retinitis (Fig. 1A). Ganciclovir administration ( $5 \mathrm{mg} / \mathrm{kg} /$ every 12 hours) was started in April 1990. Ophthalmoscopic findings showed marked improvement, and in May the dose of ganciclovir was reduced to

From the Department of Pediatrics and Child Health, *Pathology, **Ophthalmology, Kurume University School of Medicine, Kurume City 830 Received for publication August 23, 1993; Accepted for publication December 28, 1993

Reprint requests should be addressed to Dr. Shinichiro Nagamitsu, the Department of Pediatrics and Child Health, Kurume University School of Medicine, 67 Asahi-machi, Kurume 830 

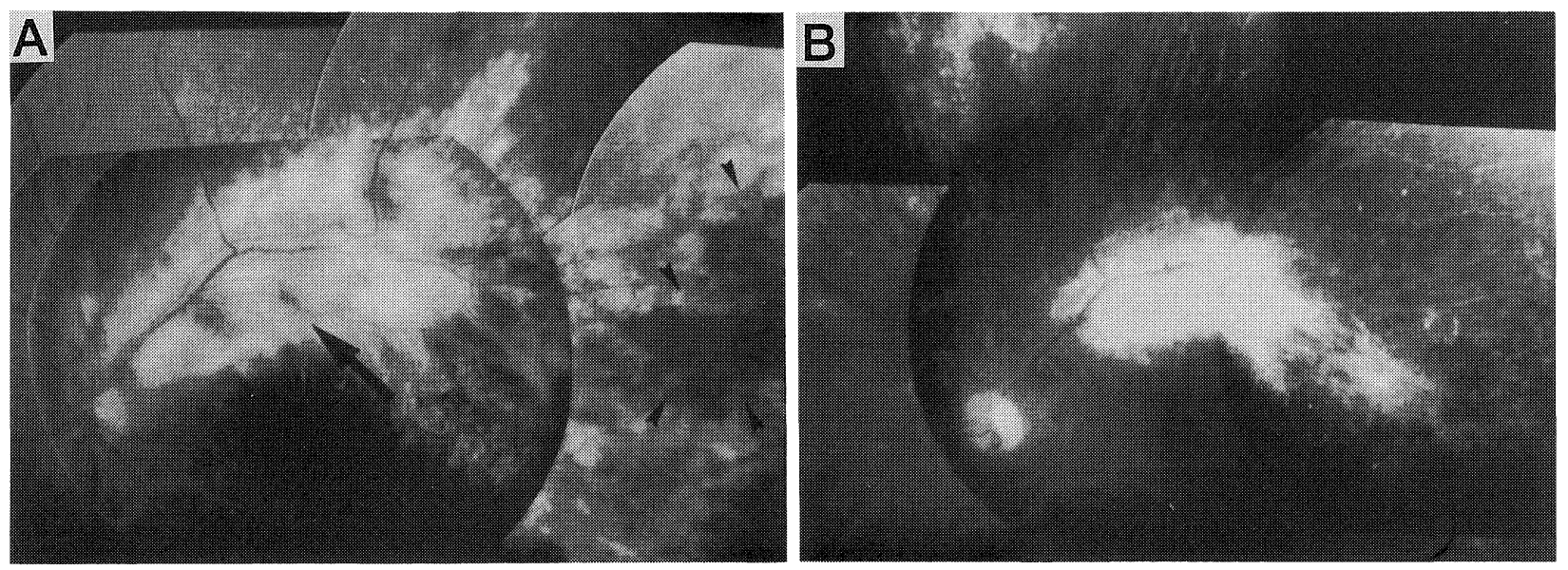

Fig. 1. Ophthalmoscopic views of left eye of acquired immunodeficiency syndrome (AIDS) patient with cytomegalovirus (CMV) retinitis. In April 1990, there is an extensive yellowish-white exudation along the retinal vasculature (arrow), with retinal hemorrhages (heads, Fig. 1A). After 6 months of ganciclovir therapy, the retinal lesions have been reduced (Fig. 1B).

$5 \mathrm{mg} / \mathrm{kg} / \mathrm{day}$. Two months later, the CMV retinitis recurred in both eyes and the patient was again administered ganciclovir, 5 $\mathrm{mg} / \mathrm{kg} / \mathrm{every} 12$ hours. He complained of headache in the right temporal and occipital areas. CT and electroencephalography (EEG) revealed no abnormalities. Sensory examination revealed hypoesthesia of touch sensation at sites corresponding to the distribution of the right maxillary branch of the trigeminal nerve. No abnormal neurological signs were present at this time. The patient's bone marrow function and immunocompetence were both severely impaired. His total lymphocyte count was $100 / \mathrm{mm}^{3}$ and the CD4 count was $5.0 / \mathrm{mm}^{3}$. Ophthalmoscopic findings remained stable for 3 months, therefore the dosage of ganciclovir was tapered to $3 \mathrm{mg} / \mathrm{kg} / \mathrm{day}$. However, the neurologic symptoms became worse, and by September the patient was disoriented and his memory was impaired. These changes were associated with the syndrome of inappropriate secretion of antidiuretic hormone (SIADH). Serum sodium was $119 \mathrm{mEq} / \mathrm{L}$, and urinary sodium was $86 \mathrm{mEq} /$ L. After the patient had recovered from SIADH, his level of consciousness was unaltered, but he developed involuntary movements and dementia. Analysis of cerebrospinal fluid (CSF) showed no changes except for a mild increase in CSF protein $(63 \mathrm{mg} / \mathrm{dl})$. Titers of antibodies in CSF were negative for HIV, CMV, toxoplasma, and cryptococcus, but the level of myelin basic protein was slightly increased $(6.1 \mathrm{ng} / \mathrm{ml}$; normal $<4 \mathrm{ng} /$ $\mathrm{ml})$. CMV virus was cultured from both urine and a throat swab. The serum CMV IgG titer was 4,800 and CMV IgM 0.08. The patient died at age 22 , four months after the onset of neurological signs, due to HIV encephalopathy and CMV infection. The brain was examined at autopsy.

\section{Ophthalmologic findings}

Ophthalmoscopic examinations were performed twice a week. After the patient complained of impairment of the visual field and of ocular floaters, his best corrected visual acuity was 0.8 in the right eye and 1.0 in the left eye. Ophthalmoscopic examination disclosed a characteristic extensive yellowishwhite exudation along the retinal vasculature, as well as retinal hemorrhages (Fig. 1A). The ophthalmoscopic findings improved markedly after ganciclovir therapy (Fig. 1B), and the patient's best corrected visual acuity was 1.0 in the right eye and 1.2 in the left eye.

\section{Neuroimaging findings}

CT was performed twice in the year preceding the patient's death. The first CT was performed when he complained of headache, the second when the consciousness disturbance appeared. The CT scans revealed progressive cerebral atrophy and abnormal low density, predominant in the front area, and moderate ventricular dilatation (Fig. 2A). Magnetic resonance imaging (MRI) was performed the day before the patient's death. MRI also revealed cerebral atrophy and ventricular dilatation. The most marked findings on MRI were diffuse highintensity areas in the white matter around the ventricles (Fig. 2B). The other significant findings were small focal or small patchy lesions which revealed high-intensity by $\mathrm{T} 2$-weighted images in the basal ganglia (Fig. 2C), midbrain (Fig. 2D), hippocampus (Fig. 2E), and the nucleus dentatus (Fig. 2F). There was no enhancing lesions with gadolinium.

\section{Histopathologic examination at autopsy}

The brain was the only organ examined at autopsy. It weighed 1,440 g. Subarachnoid hemorrhage was grossly evident on the occipital and bilateral temporal lobes. The horizontal section including frontal lobes and basal ganglia appears grossly normal (Fig. 3A). Microscopically, characteristic CMV intranuclear inclusion bodies were observed in most sites of the brain including the hippocampus (Fig. 3B), white matter, basal ganglia, midbrain, medulla oblongata, the nucleus dentatus and in the retina (Fig. 3C). Infiltration by monocyte-macrophage with multinucleated giant cells, as is characteristic of HIV encephalopathy was observed in the white matter (Fig. 3D) and 


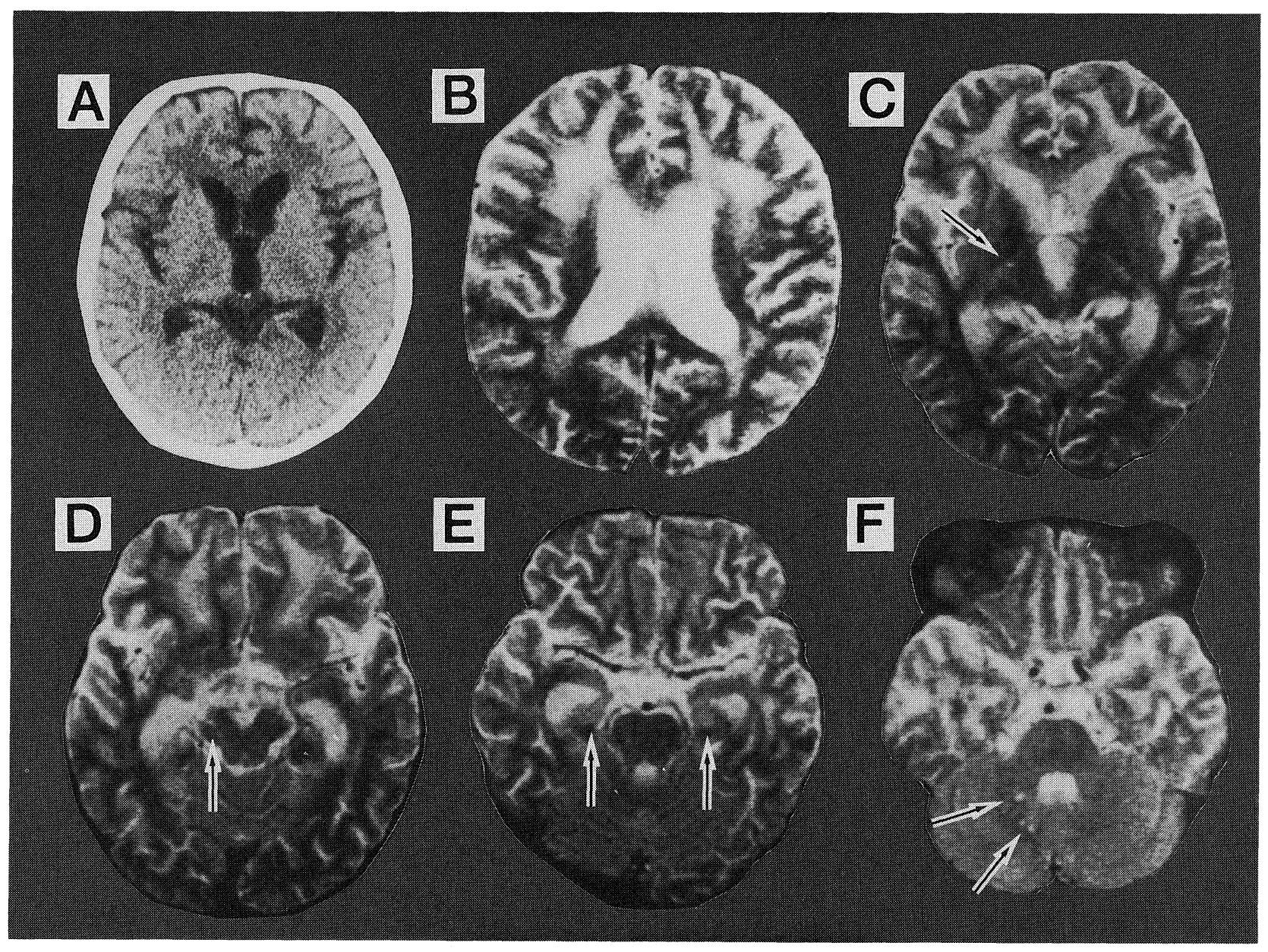

Fig. 2. Computer tomography (CT) and magnetic resonance imaging (MRI) (T2-weighted) images of the brain. CT scan shows cerebral atrophy, ventricular dilatation, and deep white matter changes in the front area (Fig. 2A). On MRI, areas of diffuse high intensity are apparent around the anterior horn (Fig. 2B). Small focal intensity (arrow) is present on the basal ganglia (Fig. 2C) and the mid brain (Fig. 2D). Small patchy lesions (arrows) are present on the hippocampus (Fig. 2E). Small focal intensities (arrows) are present around the nucleus dentatus (Fig. 2F).

hippocampus.

\section{Discussion}

AIDS was first recognized in 1980 as a syndrome of acquired immunodeficiency in young homosexual males. In Japan, about 2,500 patients are infected with HIV (1). The number of patients who develop AIDS through sexual activity is increasing. At least $60 \%$ will eventually develop neurologic symptoms; neuropathologic abnormalities are present in about $90 \%$ of such patients at autopsy (3). The most frequent cause of neurologic dysfunction is subacute encephalitis caused by the HIV infection. Neurologic disorders associated with AIDS may be caused not only by HIV infection but also by the presence of opportunistic infections and neoplasms (3). Such opportunistic infections are the major cause of death in AIDS patients. Since there is no successful treatment for AIDS, the prevention and treatment of opportunistic infection are very important in managing patients with this disease.
Cytomegalovirus is a major cause of opportunistic infection in AIDS patients. Maeda and colleagues reported that, of 14 AIDS deaths, only 2 were diagnosed as having CMV infection before death, while CMV infection was detected postmortem in 12 cases (2). The incidence of ocular manifestations in AIDS ranges from 50 to $73 \%(4,5)$. CMV retinitis was found at autopsy in 35\% AIDS patient's eyes (6). Cytomegalovirus retinitis was diagnosed in the present patient by ophthalmoscopic findings. The presence of CMV viremia was confirmed by positive urine and throat cultures. The administration of ganciclovir was highly effective in improving his retinal necrotic lesions. Although CMV retinitis recurred during his course, ganciclovir, administrated at a dose as low as $3 \mathrm{mg} / \mathrm{kg} / \mathrm{day}$, was able to control the infection.

Despite a transient improvement in the CMV infection, the patient's neurologic symptoms grew worse. Bamborschke et al reported the successful treatment of acute cytomegalovirus encephalitis with ganciclovir in non-AIDS patient (7). Price and colleagues reported that treatment of an HIV positive 

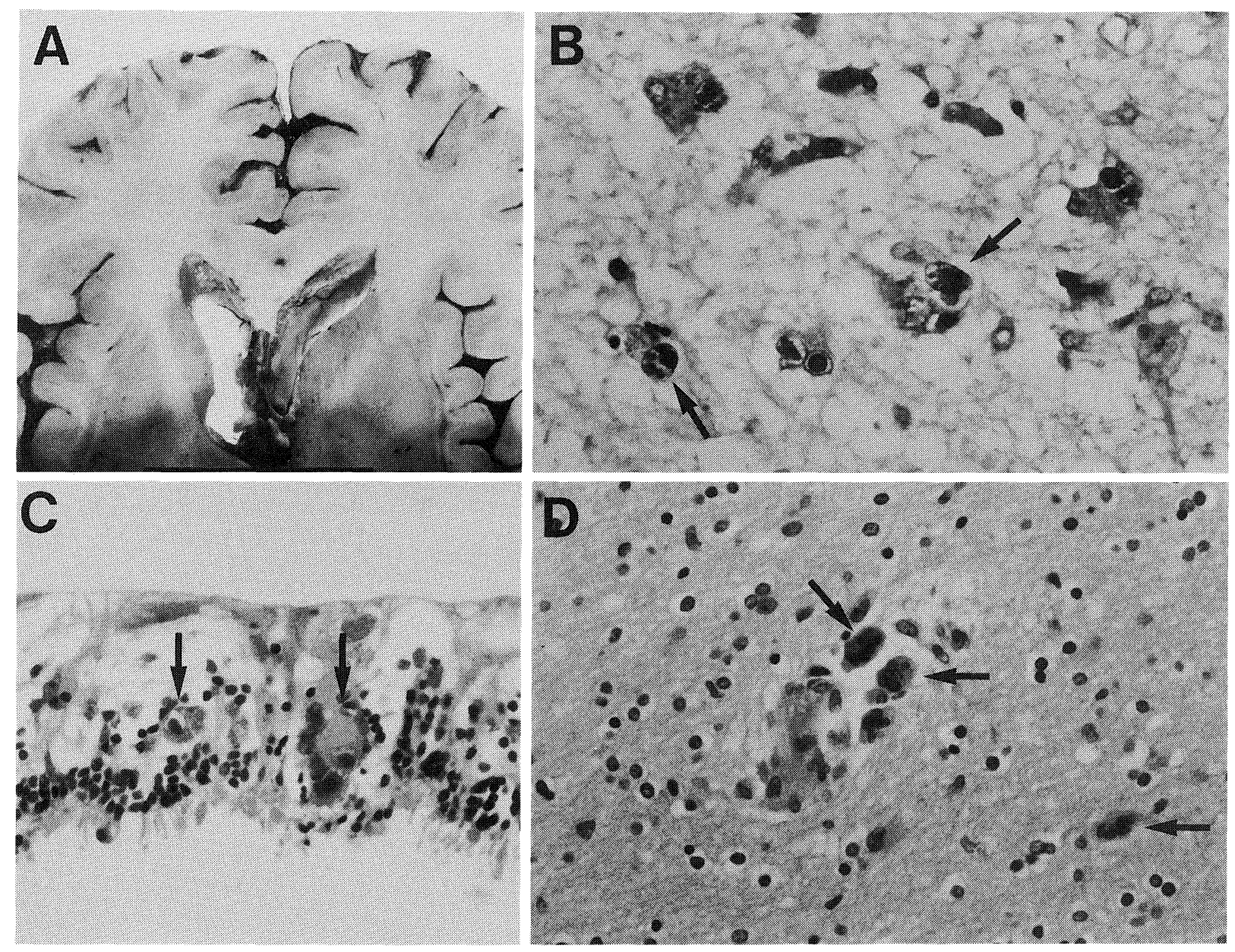

Fig. 3. Histopathologic examination of acquired immunodeficiency syndrome (AIDS) patient. Horizontal section at the level of the lateral ventricle is grossly normal (Fig. 3A). Characteristic cytomegalovirus (CMV) intranuclear inclusion bodies (arrows) are present in the hippocampus (Fig. 3B) and the retina (Fig. 3C). Multinucleated giant cells (arrows, Fig. 3D) characteristic of human immunodeficiency virus (HIV) encephalopathy are noted in the periventricular white matter (HE stain, $\times 400$ ).

patient with CMV ventriculitis with ganciclovir produced radiological improvement of the ventriculitis and cultures that were negative for CMV, yet the patient obtained little neurological benefit (8). Ganciclovir is effective in patients with CMV infection and normal immune status, but it is not always clinically effective in patients with CMV infections and who are immunodeficient, such as those with HIV. Belec et al described multinucleated giant cells, co-infected with HIV and CMV and suggested that CMV may be a co-factor in the pathogenesis of HIV encephalopathy (9).

Neuroradiologic methods such as MRI and CT scan are useful in evaluating patients with neurological complications due to AIDS. HIV encephalopathy on MRI shows diffuse patches of increased signal intensity in the deep white matter, usually bilateral, which do not involve the gray matter $(10,11)$. On the present MRI, diffuse or patchy lesions of high intensity were present in the white matter around the ventricles and hippocampus in which histopathological findings of characteristic HIV encephalopathy were observed. Focal lesions, pre- dominantly of the basal ganglia, are common in opportunistic infection such as progressive multifocal leukoencephalopathy, and toxoplasmosis (11). Detailed findings on MRI have not been described in patients with CMV encephalitis. Grafe et al reported that CMV encephalitis was seen as foci of increased signal intensity on MRI (12). On the present MRI, small, focal and patchy lesions with high intensity were present in multiple sites of the brain including the hippocampus, basal ganglia, midbrain, medulla oblongata, the nucleus dentatus, in which CMV intranuclear inclusion bodies were observed on histopathological examination. The focal or patchy high-intensity signals may have been due to the necrosis or infarction produced by CMV.

The present patient was also diagnosed as having SIADH. His serum sodium was $119 \mathrm{mEq} / \mathrm{L}$ (urinary sodium was 86 $\mathrm{mEq} / \mathrm{L}$ ) and was not associated with volume depletion or diarrhea. Agarwal et al reported that 36 of 103 patients (35\%) with AIDS who were admitted with opportunistic infection had a serum sodium of less than or equal to $130 \mathrm{mEq} / \mathrm{L}$ (13). They 


\section{Nagamitsu et al}

suggested that SIADH is a preterminal condition in some patients and may result from the severe stress brought on by opportunistic infection or by the HIV infection itself.

Opportunistic infection is the major cause of death in AIDS, so early diagnosis and treatment of such infections are very important. We conclude that in the present case, neuroimaging findings were compatible with the neuropathologic observations. Thus, MR imaging is useful in detecting the CNS lesions produced by HIV or by opportunistic infection.

\section{References}

1) Annual Meeting of Japanese Committee on AIDS Surveillance: Japanese Ministry of Health and Welfare. July 31, 1992 (in Japanese).

2) Maeda Y, Funada N, Kawaguchi K. Pathology of AIDS; anatomicopathological findings in 14 autopsy cases. Pathology and Clinic 12: 1491, 1989 (in Japanese).

3) Gabuzda DH. Neurologic disorders associated with HIV infections. J Am Acad Dermatol 22: 1232, 1990.

4) Holland GN, Pepose JS, Pettit TH, et al. Acquired immune deficiency syndrome; ocular manifestations. Ophthalmology 90: 859, 1983.

5) Freeman WR, Lerner CW, Mines JA, et al. A prospective study of the ophthalmologic findings in the acquired immune deficiency syndrome. Am J Ophthalmol 97: 133, 1984.

6) Pepose JS, Holland GN, Nestor MS, et al. Acquired immune deficiency syndrome. Ophthalmology 92: 472, 1985.

7) Bamborschke $S$, Wullen $T$, Huber $M$, et al. Early diagnosis and successful treatment of acute cytomegalovirus encephalitis in a renal transplant recipient. J Neurol 239: 205, 1992.

8) Price TA, Digioia RA, Simon GL. Ganciclovir treatment of cytomegalovirus ventriculitis in a patient infected with human immunodeficiency virus. Clin Infect Dis 15: 606, 1992.

9) Belec L, Gray F, Mikol J, et al. Cytomegalovirus (CMV) encephalomyeloradiculitis and human immunodeficiency virus (HIV) encephalitis: Presence of HIV and CMV co-infected multinucleated giant cells. Acta Neuropathol 81: 99, 1990.

10) Olsen WL, Longo FM, Mills CM, et al. White matter disease in AIDS: Findings at MR imaging. Radiology 169: 445, 1988.

11) Balakrishnan J, Becker PS, Kumar AJ, et al. Acquired immunodeficiency syndrome: Correlation of radiologic and pathologic findings in the brain. Radiographics 10: 201, 1990.

12) Grafe MR, Press GA, Berthoty DP, et al. Abnormalities of the brain in AIDS patients: Correlation of postmortem MR findings with neuropathology. AJNR 11: 905, 1990.

13) Agarwal A, Soni A, Ciechanowsky M, et al. Hyponatremia in patients with the acquired immunodeficiency syndrome. Nephron 53: 317, 1989. 